\title{
X-ray Line Profile Analysis of Nanoparticles in Proton Exchange Membrane Fuel Cell Electrodes
}

\author{
Matthias Loster, ${ }^{* \dagger} \dagger$ Davor Balzar, $\$$ K. Andreas Friedrich, ${ }^{\S}$ and Juirgen Garche \\ Center for Solar Energy and Hydrogen Research Baden-Wurttemberg, Division 3, Electrochemical Energy \\ Storage and Conversion, Helmholtzstrasse 8, 89081 Ulm, Germany
}

Received: March 7, 2007; In Final Form: May 3, 2007

\begin{abstract}
We present a method to extract X-ray diffraction patterns from a multiphase system and analyze the particle size distribution of each phase. The method is demonstrated for crystalline nanoparticles in the electrodes of proton exchange membrane fuel cells (PEMFCs), where it is particularly useful to determine particle size distributions without destroying the device. The structure of the electrodes has a considerable influence on the power and durability of a fuel cell and can be further optimized, for example with respect to the durability of the cell. Since the membrane electrode assembly (MEA) contains multiple and partially X-ray transparent layers, the individual catalyst signals from the anode (platinum-ruthenium alloy) and the cathode (platinum) can be extracted from the diffraction patterns recorded of either side of the MEA using the technique presented in this article. By analysis of the platinum (220) reflection by fitting a pseudo-Voigt function, the individual particle size distributions are determined for the anode and the cathode. The catalyst surface area loss due to particle growth is studied in long-term experiments during the operation of a single model cell for $2100 \mathrm{~h}$ and, for comparison, during the storage in different gas atmospheres $\left(\mathrm{Ar}, \mathrm{H}_{2}\right.$, and $\left.\mathrm{O}_{2}\right)$ for $6500 \mathrm{~h}$. With respect to the single cell operation, approximately one-third of the surface is lost in the storage experiment with a slight influence from the gas atmosphere and the catalyst type. The comparison with transmission electron micrographs shows that the size distributions have a similar shape and width but differ in absolute sizes.
\end{abstract}

\section{Introduction}

Small particles with dimensions of several nanometers are used in a variety of technological applications. The usually complex systems consist of different parts that interact and cannot function individually. With conventional methods like transmission electron microscopy or powder X-ray diffraction, it is often difficult or even impossible to study the small particles in operando. In this article, we present a new method to characterize individual particle ensembles that are part of a device, allowing nondestructive studies close to "in situ" operating conditions.

Proton exchange membrane fuel cells (PEMFCs) are an example of such devices. Their performance has improved significantly through recent research and development efforts so that the commercialization in mass markets is anticipated soon. Some problems remain, however, like the insufficient durability and the high cost of the catalysts and the membrane. At this stage, it is desirable to improve the understanding of the fundamental processes, in particular those leading to performance degradations. Different methods have been applied to characterize the porous mixture of nanometer-sized catalyst particles, carbon support, and polymer electrolyte in PEMFCs. Among these methods are the imaging with scanning and transmission electron microscopy, X-ray diffraction, and X-ray

* To whom correspondence should be addressed. Phone: (510) 6420190. Fax: (510) 643-8793. E-mail: ml@loster.com.

† Department of Physics, University of California, Berkley, CA 94720.

Department of Physics and Astronomy, University of Denver, Denver, CO 80208 and National Institute of Standards and Technology, Boulder, CO 80305.

$\S$ German Aerospace Center, Electrochemical Energy Technology, Pfaffenwaldring 38-40, 70569 Stuttgart, Germany. absorption spectroscopy. ${ }^{1,2} \mathrm{X}$-rays, in general, allow the sampling of a large volume with many particles while, in contrast, electron microscopy provides only data of a very small section of the electrode. A wide X-ray beam can therefore capture up to $10^{11}$ times more particles than an electron microscope. Usually, small parts of the electrode need to be removed as specimens, but methods have started to develop recently that allow the investigation in "in situ" conditions. ${ }^{3-5}$ Because the presence of fuel gases is required during operation, it is often better to use X-rays for which the scattering probability at the gas molecules is significantly lower than for electron beams. However, when using methods with a high penetrability, it is necessary to separate the signals of the components, in particular those from the two electrodes under investigation. This can be done, for example, by using entirely different materials on each electrode and examining only their specific signatures. If materials with a similar structure like platinum and platinumruthenium alloys are to be examined, a different approach is needed. For X-ray diffraction measurements, where the beam is usually reflected by the specimen, each electrode and the intermediate electrolyte contribute to the diffraction pattern resulting in overlapping signals.

The method introduced in this article facilitates the separation of the individual component signals and allows one to follow the changes of the catalyst particles independently on each electrode. In a long-term experiment with a single model cell, the platinum (220) peak is evaluated to calculate the particle size distributions and the surface area loss on both electrodes before and after the operation. The results are compared with the size distributions from transmission electron micrographs of samples that were removed from the electrodes. The exposure of identical electrodes to argon and typical fuel gases, hydrogen 
and oxygen, for a prolonged period of time at elevated temperatures shows approximately one-third of the surface area is lost as compared to the operation in the model cell. In contrast to the surface loss that can be determined with electrochemical methods, the X-ray based analysis provides insight into a mechanism that is believed to be relevant for the nonreversible performance degradation of the cells.

\section{Experimental Section}

MEA Production. Two identical MEAs with an effective electrode area of $50 \times 50 \mathrm{~mm}^{2}$ each were produced by spray coating a Nafion 105 membrane with the highly disperse carbon supported catalyst materials from Heraeus, $40 \mathrm{wt} \%$ platinumruthenium alloy on the anode and $40 \mathrm{wt} \%$ platinum on the cathode. The projected metal loadings were $0.35 \mathrm{mg} / \mathrm{cm}^{2}$ on the anode and $0.40 \mathrm{mg} / \mathrm{cm}^{2}$ on the cathode. The dry Nafion content was $50 \%$ on both electrodes. The pretreatment and coating were performed according to a previously published procedure.

Exposure to Operating Conditions. The first MEA prepared with carbon supported catalysts was exposed to fuel cell operating conditions in a single cell test stand and investigated before and after long-term operation for $2100 \mathrm{~h}$. The MEA was placed between two Toray TGP 60 carbon paper gas diffusion layers impregnated with 25 wt $\%$ Teflon to improve water management. The cell fixture was acquired from ElectroChem, Inc. and had graphite serpentine type flow fields and external heating pads to keep the cell temperature at $60{ }^{\circ} \mathrm{C}$. Hydrogen and oxygen gases with $99.9 \%$ purity were supplied continuously from sufficiently large on-site tanks for uninterrupted operation. The reactant gases were heated, humidified, and routed to the electrodes at a controlled flow rate under ambient pressure. Bubble humidifiers that were heated to $60{ }^{\circ} \mathrm{C}$ provided close to $100 \%$ relative humidification. Anode and cathode reactant stoichiometries were kept at $\lambda_{\mathrm{a}}=1.3$ and $\lambda_{\mathrm{c}}=2$, respectively. The electric load was set to a constant current density of 0.4 $\mathrm{A} / \mathrm{cm}^{2}$. After the long-term tests were ended, the cell was dismantled and the MEA was prepared for analysis. Because of the mechanical compression and heating of the cell, the gas diffusion layers were usually sticking to the MEA. To avoid damage to the electrode, the gas diffusion layers were cautiously peeled off after immersion in ultrapure water for approximately 15 min. The second, identical, MEA was cut to pieces of approximately $10 \times 30 \mathrm{~mm}^{2}$. These fragments were labeled and sealed in glass cylinders that were, after evacuating the air, filled from gas tanks to atmospheric pressure with argon $(99.996 \%$ purity), hydrogen ( $99.999 \%$ purity), and oxygen $(99.999 \%$ purity) gases. The cylinders were then stored in a temperature controlled furnace for $6500 \mathrm{~h}$, with $2900 \mathrm{~h}$ at $40{ }^{\circ} \mathrm{C}$ and 3600 $\mathrm{h}$ at $70^{\circ} \mathrm{C}$. Afterward, the glass cylinders were removed from the furnace and opened to retrieve the MEA fragments for further investigations by X-ray diffractometry.

X-ray Diffractometer. A Bruker AXS D8 DISCOVER with a general area detector diffraction system (GADDS) area detector, a copper $\mathrm{K} \alpha$ source, and a single Göbel mirror was operated at $40 \mathrm{kV}$ and $40 \mathrm{~mA}$. A significant advantage of this instrument is that a certain angular range, known as a frame, can be recorded at once with the help of the parallel beam and the area detector. In contrast to the more commonly used Bragg-Brentano instruments, the sampling time is thereby significantly reduced. Diffraction patterns were recorded of entire MEAs after removing the gas diffusion layers and fixing them with clamps on the sample stage to prevent swelling movements. A flat single-crystal silicon plate cut at its (911)

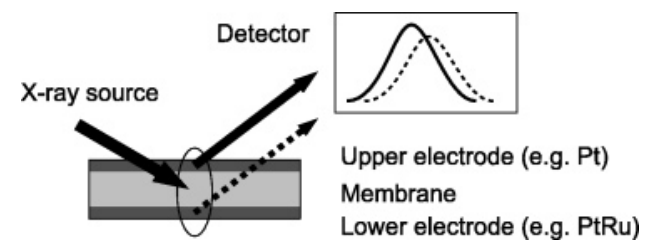

Figure 1. Sketch of the measurement principle. The source beam is diffracted at the upper and at the lower electrodes, thus producing a diffraction pattern that is an overlay of signals from both electrodes. When the MEA is turned around, i.e., the upper and the lower electrodes are swapped, a second diffraction pattern is obtained that allows the separation of the individual electrode patterns.

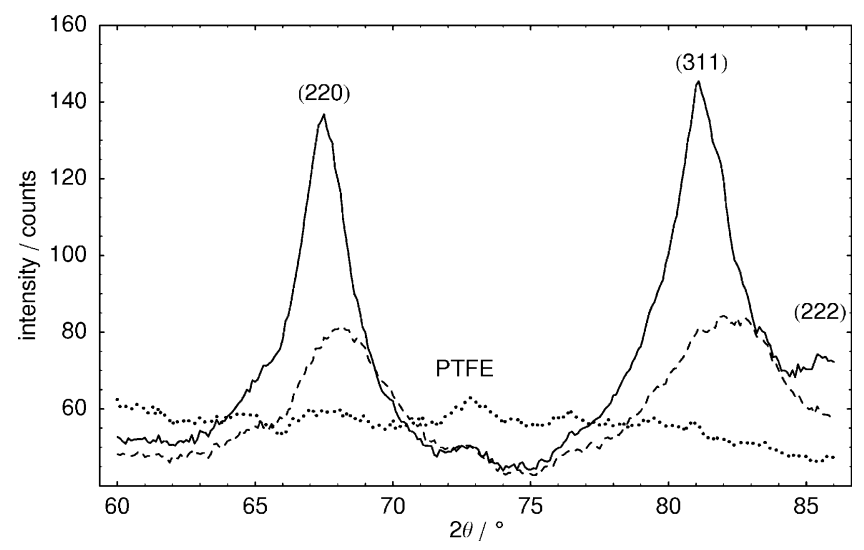

Figure 2. X-ray diffraction patterns of electrode components: $40 \mathrm{wt}$ $\% \mathrm{PtRu} / \mathrm{C}$ (dashed), 40 wt \% Pt/C (continuous), Nafion 105 (dotted). A distinct PTFE peak is marked, and the Miller indices of the reflecting crystal planes are indicated for Pt. The angle of incidence of the parallel beam is $\theta_{1}=30^{\circ}$.

plane was placed under the sample to shield the aluminum stage. The frames shown in this article span a $2 \theta$ range from $60^{\circ}$ to $86^{\circ}$ and required a sampling time of $30 \mathrm{~min}$.

Transmission Electron Microscope. A Philips CM-20 Super-Twin transmission electron microscope with $200 \mathrm{keV}$ beam energy and a point resolution of $0.24 \mathrm{~nm}$ was available for the experiments. The electrode samples were prepared by scraping off a part of the electrode layer. The scrapings were suspended in ethanol and ultrasonicated to create homogeneous slurries that were deposited onto a carbon microgrid on which the catalyst particles were imaged with the microscope.

\section{Component Signal Separation}

The method presented in this article allows the extraction of individual component signals from the diffraction patterns of the entire layered structure. In particular, each component used during the MEA production is recorded separately, i.e., the platinum-ruthenium alloy, the platinum catalyst, and the Nafion polymer electrolyte. A linear combination of the component patterns, if multiplied with the correct coefficients, will fit the patterns recorded of either side of the MEA. Equally, two component signals can be extracted from the data of the MEA if the coefficients are known. Figure 1 shows schematically how the signals from both electrodes overlap in the diffraction pattern of the MEA. It is presumed that the coefficients and the remaining components do not change during operation.

Component Diffraction Patterns. Diffraction patterns of the platinum-ruthenium alloy and the platinum catalyst, both supported on carbon, and the Nafion membrane are shown in Figure 2. For the analysis, it will be important that the illuminated sample area and the penetration depth are constant over the displayed angular range because a parallel beam instrument is used. 
The diffraction pattern of platinum is consistent with a face centered cubic (fcc) crystal lattice and is in agreement with the ICDD powder diffraction database. In the diffraction pattern, the peaks are substantially broadened due to the small crystallite sizes. The (111) and (200) reflections, not visible in the figure, are partially overlapping. The (311) reflection overlaps with the (222) peak, partly visible toward higher angles in Figure 2. Thus, the (220) peak will be used in the subsequent fitting procedures since it is not overlapping with other reflections. The platinumruthenium alloy has reflections similar to platinum, although they are shifted toward slightly higher values of $2 \theta$. This shift indicates that the alloy is formed with a platinum lattice that has a slightly smaller lattice constant. ${ }^{6,7}$ No evidence for metallic ruthenium or ruthenium oxides is found, since reflections at those angles are absent.

The small peak at $72.6^{\circ}$ is the result of a small amount of polytetrafluoroethylene (PTFE) that was added during catalyst production to increase the hydrophobicity. The powder diffraction database lists major reflections of PTFE (no. 47-2217) at $2 \theta=65.9^{\circ}$ and $72.6^{\circ}$. The latter is also visible in the diffraction pattern of Nafion, while the first peak appears only as a small disturbance of the (220) reflection in the catalyst materials. The relatively small background stems from amorphous contributions in the carbon support and Nafion.

Fitting the Diffraction Patterns of a MEA. The diffraction pattern of a MEA is a linear combination of the component intensities. The coefficients describe the contribution of each component depending on the geometric arrangement. For example, when the anode is facing the beam, a strong platinumruthenium signal and a weak platinum signal are expected, and vice versa when the MEA is turned around. The polymer membrane between the electrodes is equally accessible from both sides; thus, its signal intensity is not expected to change significantly. Since the diffraction patterns of the components are already known, their linear combination, i.e., the weighted sum of the intensities, can be fitted to the patterns of either side of the MEA. Therefore, the detected intensity $I_{i \text {,mea }}$ is described by the sum of the component intensities $I_{i, \text { sum }}$ for every angle $2 \theta$ (eq 1). In eq $1, i$ is the side of the MEA facing the

$$
I_{i, \text { mea }} \approx I_{i, \text { sum }}=\sum a_{i, j} I_{j}=a_{i, 1} I_{1}+a_{i, 2} I_{2}+a_{i, 3} I_{3}
$$

beam ( $\mathrm{a}=$ anode, $\mathrm{c}=$ cathode $), j$ is the component index $(1=$ $\mathrm{PtRu}, 2=\mathrm{Pt}, 3=$ Nafion), $I_{j}$ is the intensity of each component, and $a_{i, j}$ is the weighting factor.

The diffraction patterns of a MEA recorded after production are shown with the anode side facing the beam $\left(I_{\mathrm{a} \text {,mea }}\right.$; see Figure 3 ) and the cathode side facing the beam $\left(I_{c, \text { mea }}\right.$; see Figure 4$)$. Displayed in the same figures are the individual component intensities weighted with factors obtained from a least-squares fit, i.e., $a_{i, j} I_{j}$, and the sums of the three weighted component intensities $I_{i, \text { sum }}$ according to eq 1 . It is noted that $I_{i \text {,sum }}$ follows closely the data obtained for the MEA, $I_{i, \text { mea }}$, indicating that the fit agrees well. Because the different component signals are not linearly dependent, the $a_{i, j}$ values are uniquely determined. The resulting $a_{i, j}$ values are listed in Table 1, giving the contributions of each component. The signal intensity of the electrode facing the beam is three times higher than that of the opposite electrode, i.e., $a_{\mathrm{a}, 1} / a_{\mathrm{a}, 2} \approx a_{\mathrm{c}, 2} / a_{\mathrm{c}, 1} \approx 3$, confirming the expectations.

Signal Extraction. With knowledge of the $a_{i, j}$ values, the diffraction intensities of the individual components, $I_{j}$, can be extracted from the recorded data $I_{i \text {,mea. }}$. As shown above, $I_{i \text {,mea }}$ is approximated by the linear combination of components $I_{i \text {,sum }}$. The number of equations is limited to the number of available

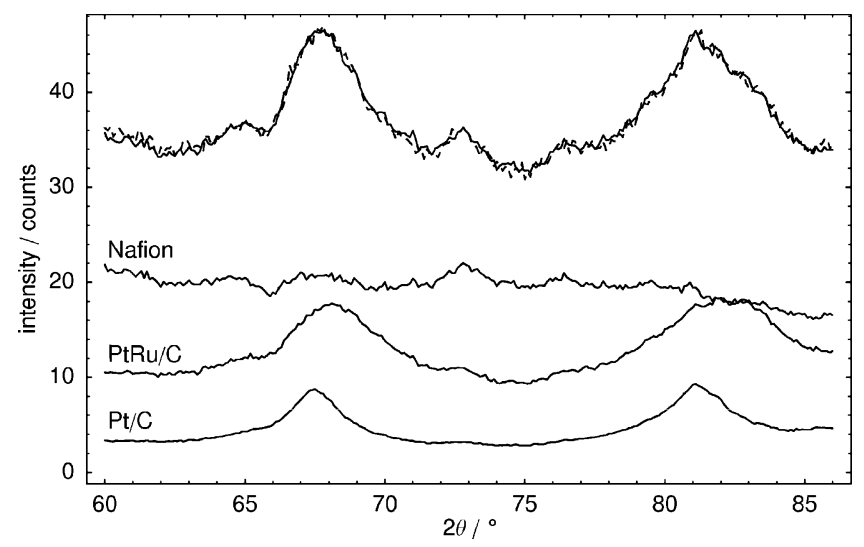

Figure 3. Fit of a linear combination of the component intensities ( $I_{\mathrm{a}, \text { sum }}$; continuous line) to the diffraction pattern of the MEA with the anode facing the beam $\left(I_{\mathrm{a} \text {,mea }}\right.$; dashed line). The component patterns are shown below, scaled with the factors obtained from the fit $\left(a_{\mathrm{a}, j} I_{j}\right.$; see Table 1).

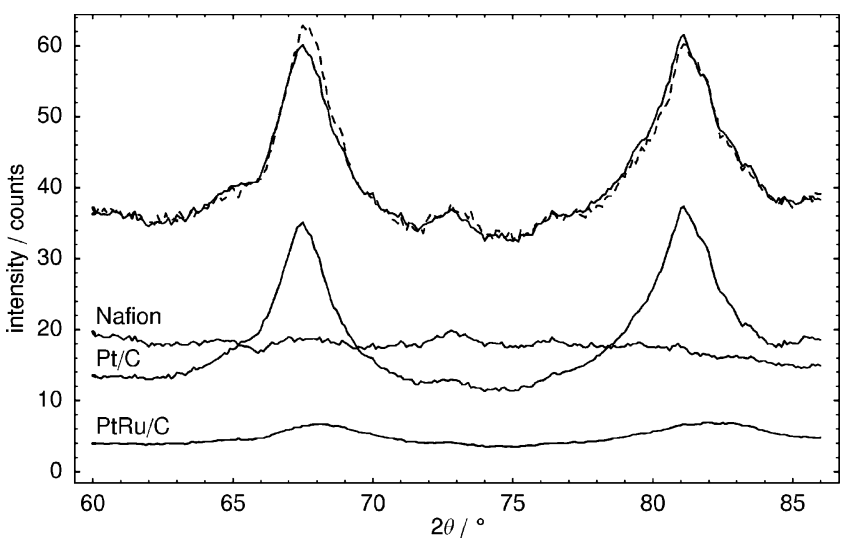

Figure 4. Component intensities $\left(I_{\mathrm{c}, \text { sum }}\right.$; continuous line) fitted to the diffraction pattern of a MEA with its cathode facing the beam $\left(I_{\mathrm{c}, \text { mea }}\right.$; dashed line). See also Figure 3.

TABLE 1: Coefficients $a_{i, j}$ Determined by Fitting $I_{\mathrm{a}, \text { sum }}$ and $I_{\mathrm{c}, \text { sum }}$ to the Diffraction Patterns of the Anode $\left(I_{\mathrm{a}, \mathrm{mea}}\right)$ and the Cathode $\left(I_{c, \text { mea }}\right)$ Sides of the MEA ${ }^{a}$

\begin{tabular}{lccc}
\hline & \multicolumn{3}{c}{$a_{i, j}$} \\
\cline { 2 - 4 } & PtRu (1) & Pt (2) & Nafion (3) \\
\hline anode (a) & 26 & 8 & 42 \\
cathode (c) & 10 & 31 & 38
\end{tabular}

${ }^{a}$ See also Figures 3 and 4.

diffraction patterns, i.e., the number of accessible sides of the MEA. Thus, two unknown component signals can be determined, in the present case, those of the platinum-ruthenium and the platinum catalysts. This is possible because the $a_{i, j}$ values are known and the intensities of Nafion, $I_{3}$, are known from the component diffraction patterns. Thus, the solution for $I_{1}$ and $I_{2}$ is given by

$$
\begin{aligned}
& I_{1}=\frac{a_{\mathrm{c}, 1} I_{\mathrm{a}, \text { sum }}-a_{\mathrm{a}, 1} I_{\mathrm{c}, \mathrm{sum}}-\left(a_{\mathrm{a}, 3} a_{\mathrm{c}, 1}-a_{\mathrm{a}, 1} a_{\mathrm{c}, 3}\right) I_{3}}{a_{\mathrm{a}, 2} a_{\mathrm{c}, 1}-a_{\mathrm{a}, 1} a_{\mathrm{c}, 2}} \\
& I_{2}=\frac{-a_{\mathrm{c}, 2} I_{\mathrm{a}, \mathrm{sum}}+a_{\mathrm{a}, 2} I_{\mathrm{c}, \mathrm{sum}}+\left(a_{\mathrm{a}, 3} a_{\mathrm{c}, 2}-a_{\mathrm{a}, 2} a_{\mathrm{c}, 3}\right) I_{3}}{a_{\mathrm{a}, 2} a_{\mathrm{c}, 1}-a_{\mathrm{a}, 1} a_{\mathrm{c}, 2}}
\end{aligned}
$$

Applying this method to a newly prepared MEA is not overly informative because the component signals could as well be obtained by just recording a diffraction pattern of the pure materials. However, after the MEA is exposed to conditions 
that induce changes in the catalyst structure, component diffraction patterns could only be obtained by removing the materials and thus destroying the MEA. Therefore, the described method avoids the destruction and allows the detection of changes in the catalyst structure while the MEA remains usable, provided that the $a_{i, j}$ and the Nafion signal remain constant over the period of operation.

\section{Line Profile Analysis}

In the following, a pseudo-Voigt function is fitted to the platinum (220) peak using a numerical procedure in the commercial software package Mathematica that offers nonlinear least-square fits with the quasi-Newton algorithm. The obtained parameters contain information about the particle size distribution and the specific surface area, as will be briefly shown in the following. The fit function is given by

$$
f_{\mathrm{fit}}(x)=a f_{\mathrm{pV}}\left(x-x_{0}\right)+b x+c
$$

where $f_{\mathrm{pV}}$ is the pseudo-Voigt function, a linear combination of a Gaussian and Lorentzian functions,

$$
f_{\mathrm{pV}}\left(x ; \gamma_{\mathrm{g}}, \gamma_{1}\right)=\gamma_{\mathrm{g}}\left[(1-\eta) f_{\mathrm{g}}(x)+\eta f_{1}(x)\right]
$$

with the mixing parameter $\eta{ }^{8}$ For the evaluation of the crystallite sizes, it is necessary to convert the fit parameters $\gamma_{\mathrm{g}, \mathrm{l}}$ to values for the integral breadths $\beta_{\mathrm{g}, \mathrm{l}}$ in reciprocal space (units of $\mathrm{nm}^{-1}$ ):

$$
\beta_{\mathrm{g}, 1}=\frac{\gamma_{\mathrm{g}, 1}}{\lambda} \cos \left(\frac{2 \theta}{2}\right)
$$

where $\lambda$ is the wavelength of the X-rays. The Fourier coefficients $A(L)$ and their second derivative are derived from the integral breadths of the Voigt function, $\beta_{\mathrm{g}}$ and $\beta_{\mathrm{l}}:^{9}$

$$
\begin{gathered}
A(L)=\exp \left(-2 L \beta_{1}-\pi \mathrm{L}^{2} \beta_{\mathrm{g}}{ }^{2}\right) \\
\frac{\mathrm{d}^{2} A(L)}{\mathrm{d} L^{2}}=\left[\left(2 \pi \mathrm{L}{\beta_{\mathrm{g}}}^{2}+2 \beta_{1}\right)^{2}-2 \pi \beta_{\mathrm{g}}{ }^{2}\right]
\end{gathered}
$$

where $L=n\left|a_{3}\right|$ are the lengths of columns of cells along the $a_{3}$ direction normal to the diffracting planes. For both, the areaweighted and the volume-weighted cases, the column-length distribution functions, $p_{\mathrm{s}}(L)$ and $p_{\mathrm{v}}(L)$ can be expressed as functions of $L$.

$$
\begin{gathered}
p_{\mathrm{s}}(L) \propto \frac{\mathrm{d}^{2}}{\mathrm{~d} L^{2}} A(L) \\
p_{\mathrm{v}}(L) \propto L \frac{\mathrm{d}^{2}}{\mathrm{~d} L^{2}} A(L)
\end{gathered}
$$

The respective mean values for the area-weighted and volumeweighted column lengths are given by

$$
\begin{gathered}
\langle L\rangle_{\mathrm{s}}=\frac{1}{2 \beta_{1}} \\
\langle L\rangle_{\mathrm{v}}=\frac{\exp \left(k^{2}\right)}{\beta_{\mathrm{g}}} \operatorname{erfc}(k)
\end{gathered}
$$

with $k=\beta_{\mathrm{l}} /\left(\pi \beta_{\mathrm{g}}\right)$.

A measure for the domain sizes is obtained by assuming a certain shape for the particles and a lognormal size distribution.
For a spherical shape, $\langle L\rangle_{\mathrm{s}}$ and $\langle L\rangle_{\mathrm{v}}$ are functions of the second, third, and fourth moments of the size distribution:

$$
\begin{aligned}
& \langle L\rangle_{\mathrm{s}}=\frac{2}{3} M_{3} / M_{2} \\
& \langle L\rangle_{\mathrm{v}}=\frac{3}{4} M_{4} / M_{3}
\end{aligned}
$$

with the $j$ th moments $M_{j}$. For the lognormal distribution

$$
P_{\ln }(x)=\frac{1}{S \sqrt{2 \pi} x} \exp \left[\frac{-(\ln x-M)^{2}}{2 S^{2}}\right]
$$

the parameters of the distribution are then given by

$$
\begin{aligned}
M & =\frac{1}{2}\left[7 \ln \left(\frac{3}{2}\langle L\rangle_{\mathrm{s}}\right)-5 \ln \left(\frac{4}{3}\langle L\rangle_{\mathrm{v}}\right)\right] \\
S & =\sqrt{-\ln \left(\frac{3}{2}\langle L\rangle_{\mathrm{s}}\right)+\ln \left(\frac{4}{3}\langle L\rangle_{\mathrm{v}}\right)}
\end{aligned}
$$

and the mean of the lognormal distribution, i.e., the mean particle diameter, is

$$
\langle P\rangle_{\mathrm{xrd}}=\exp \left(M+\frac{S^{2}}{2}\right)
$$

The specific surface area $S_{\mathrm{xrd}}$ of the catalyst, i.e., the surface area $A$ divided by the density of platinum $\rho=21.45 \mathrm{~g} / \mathrm{cm}^{3}$ and the volume $V$, can be calculated assuming spherical particles and a lognormal distribution of the particle diameters. Thus,

$$
S_{\mathrm{xrd}}=\frac{A}{\rho V}=\frac{4 \pi \sum r_{i}^{2}}{\left({ }^{4} / 3\right) \rho \pi \sum r_{i}^{3}}=\frac{3}{\rho} \frac{M_{2}}{M_{3}}
$$

with the second and third raw moments of the lognormal distribution being

$$
\begin{aligned}
& M_{2}=\exp \left[2\left(M+S^{2}\right)\right] \\
& M_{3}=\exp \left[3 M+\frac{9}{2} S^{2}\right]
\end{aligned}
$$

\section{Results}

In the following, the $\mathrm{Pt}(220)$ peaks in the diffraction patterns of the tested MEAs are analyzed. The fit function as given by eq 3 includes a linear background, and the peak center is defined by $x_{0}$. All fits were restricted to angles ranging from $2 \theta=60^{\circ}-$ $72^{\circ}$, covering the broad (220) reflection and avoiding the influence of the small peak attributed to PTFE at approximately $72.6^{\circ}$. The starting values were adjusted to allow convergence of the fit, always letting $b=0, x_{0}=67.6^{\circ}$, and $1 \leq \beta_{\mathrm{g}}, \beta_{1} \leq 2$.

Long-Term Operation in a Single Cell. The first MEA was exposed to a true fuel cell environment in a single cell test stand that allowed continuous operation under constant conditions over $2100 \mathrm{~h}$. A constant current of $0.4 \mathrm{~A} / \mathrm{cm}^{2}$, or $10 \mathrm{~A}$, was drawn over the entire period. The cell voltage is shown in Figure 5 as a function of the operating time. Three interruptions due to infrastructure changes in our laboratory occurred during this period, the first one at $180 \mathrm{~h}$ and two longer ones for less than 4 days each at 790 and $1720 \mathrm{~h}$. After two of these interruptions, the first and the third ones, the cell voltage increased significantly by $30-40 \%$, a known behavior that is called recovery after reversible performance degradation. The reversible power loss can be caused, for example, by liquid product water that 


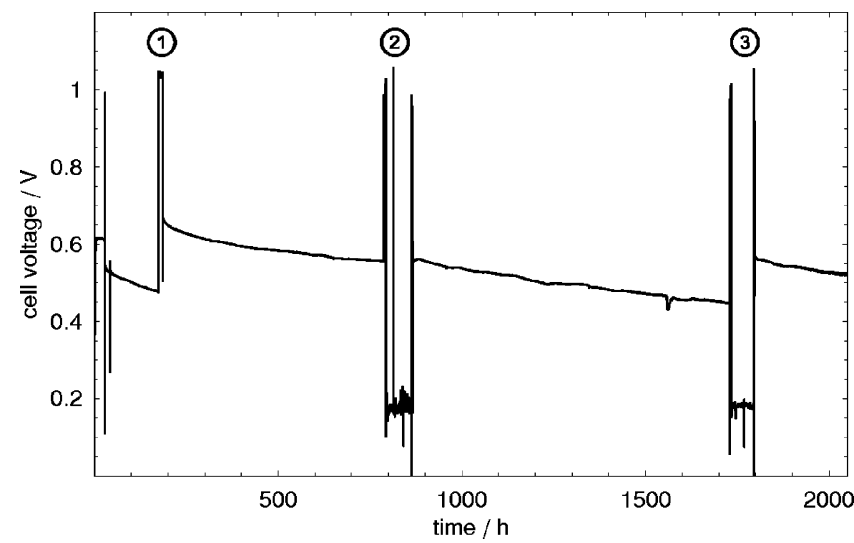

Figure 5. Cell voltage transient during the long-term experiment with a constant load of $0.4 \mathrm{~A} / \mathrm{cm}^{2}(10 \mathrm{~A})$. Three interruptions occurred during the operation that are marked with numbers in circles (see text for details).

blocks the porous gas diffusion layers or changes the membrane properties. An interruption of the operation stops the water production, and the cell may dry and recover to a higher performance level. In addition, also an irreversible performance degradation is observed that cannot be recovered by operational changes. Possible causes are the aging and decomposition of the membrane, increase of contact resistances due to corrosion, loss of hydrophobicity in the diffusion layers, and in particular, the irreversible growth of catalyst particles that is studied here. The maximum voltage under load is reached at $0.65 \mathrm{~V}$ after a running-in period of $180 \mathrm{~h}$, immediately after the first interruption. The mean reversible voltage degradation until the beginning of the third interruption is $147 \mu \mathrm{V} / \mathrm{h}$, while the irreversible (nonrecoverable) degradation measured till after the end of the third interruption is $62 \mu \mathrm{V} / \mathrm{h}$. This corresponds to a power loss at constant current of approximately $20 \%$ over the entire operating period.

Diffraction patterns of the MEA were recorded immediately before and after the operation in the test stand. Figure 6 shows, for comparison, the patterns of the unused carbon supported catalysts platinum-ruthenium and platinum and the catalyst component signals extracted from MEA recordings before and after the experiment. The signal-to-noise ratio of the extracted data is notably smaller than that of the unused catalysts because less material is examined in the comparatively thin electrodes. However, the quality is sufficient for the line profile analysis with a pseudo-Voigt function.

The peaks shown in Figure 6 have similar widths in the first two rows and become narrower in the third row for both, anode and cathode. That is, the peak widths of the unused catalyst are similar to the widths of the peaks extracted from the MEA before the exposure to operating conditions. After the experiment, their widths decrease notably, indicating a growth of the catalyst particles that is more emphasized on the cathode.

A more detailed analysis is performed by fitting pseudo-Voigt functions to the line profiles. The fitted functions are also shown in Figure 6, agreeing well with the experimental data in all six cases. Table 2 lists the parameters of the pseudo-Voigt functions, i.e., the Gaussian and Lorentzian components of the integral breadths, $\beta_{\mathrm{g}}$ and $\beta_{\mathrm{l}}$. The ratio of the volume-weighted to the area-weighted mean column lengths fulfills the inequality 1.31 $\left\langle\langle L\rangle_{\mathrm{v}} /\langle L\rangle_{\mathrm{s}}<2.00\right.$, thereby indicating the correct estimation of the background. ${ }^{9}$ The mean particle sizes $\langle P\rangle_{\mathrm{xrd}}$ are calculated from the pseudo-Voigt function parameters. The mean particle sizes determined from TEM images, $\langle P\rangle_{\text {tem }}$, show the result of averaging approximately 400 randomly selected particles in images obtained from catalyst materials scraped off the electrodes. $\langle P\rangle_{\mathrm{xrd}}$ is smaller than $\langle P\rangle_{\text {tem }}$ for all samples, indicating a systematic discrepancy between the two methods. The specific surface area $S_{\mathrm{xrd}}$ is derived from the line profile parameters that allow one to compute the second and third moment of the size distribution. For all data derived from the line profile, it is assumed that the particles have a spherical shape and have a lognormal size distribution. A summary of the relative changes during the operation is shown in Table 3, i.e., particle sizes and the specific surface areas. The growth of the mean particle diameter observed with XRD is considerably higher than that observed in the TEM images for both electrodes, a fact that will be discussed in detail in the next section.

The size distributions obtained with XRD and TEM are compared in Figure 7. The continuous lines show the lognormal distributions whose parameters are calculated from those of the pseudo-Voigt function. For comparison, the bar charts show the size distributions obtained by evaluating approximately 400 particles for each sample in transmission electron micrographs. As in Figure 6, the results for the anode and for the cathode are shown in the left and right columns, respectively. From the top, the data of the unused catalysts, the MEA before operation, and the MEA after operation are shown in each row. The same TEM-based data is displayed for the unused catalysts and the electrodes before operation. In contrast, the data set based on line profile analysis shows the unused catalysts and the catalysts in the MEA obtained in separate experiments. The small particle growth observed here probably arises from the spray coating process, where catalyst ink is transferred on the hot membrane. In general, the particle sizes determined from line profile analysis are smaller by $0.3-0.8 \mathrm{~nm}$ than those determined from the TEM images.

Exposure to Different Gas Atmospheres. A similar analysis is performed for the three identical MEA fragments that were exposed to argon, hydrogen, and oxygen atmospheres for a total of $6500 \mathrm{~h}$. Both MEAs, the one used in the single cell experiment and the one serving as a source for the fragments, were produced to be identical. Therefore, the losses of surface area can be compared relative to each other.

The integral breadth parameters determined from the fits, $\beta_{\mathrm{g}}$ and $\beta_{\mathrm{l}}$, are listed in Table 4 together with the ratio of the volumeweighted to the area-weighted mean column lengths, the mean crystallite sizes, and the specific surface areas. The values of $\langle L\rangle_{\mathrm{v}} /\langle L\rangle_{\mathrm{s}}$ indicate the correct estimation of the X-ray background. The specific surface areas relative to their initial values (i.e., the values $S_{\text {xrd }}$ for "before, anode" and "before, cathode" in Table 2) are listed in Table 5 for each gas atmosphere and for each electrode of the MEA. While the surface area loss is always higher than $19 \%$ for all electrodes and atmospheres, the loss is especially pronounced on the cathode in the hydrogen atmosphere and on the anode in the oxygen atmosphere. Generally, the area loss on the anode is higher than on the cathode.

\section{Discussion}

Although it is possible to determine the catalyst particle sizes very accurately with electron microscopy, there is a need for a method that allows "in situ" measurements. First, the removal of electrode material from the MEA can change the structure of the sample and the MEA. When several investigations of the same specimen are to be performed over the duration of a single experiment, the technique should especially have no impact on the sample. Second, the evaluation of micrographs is limited to a relatively small number of particles, determined by the time needed for preparation and imaging. This prevents 

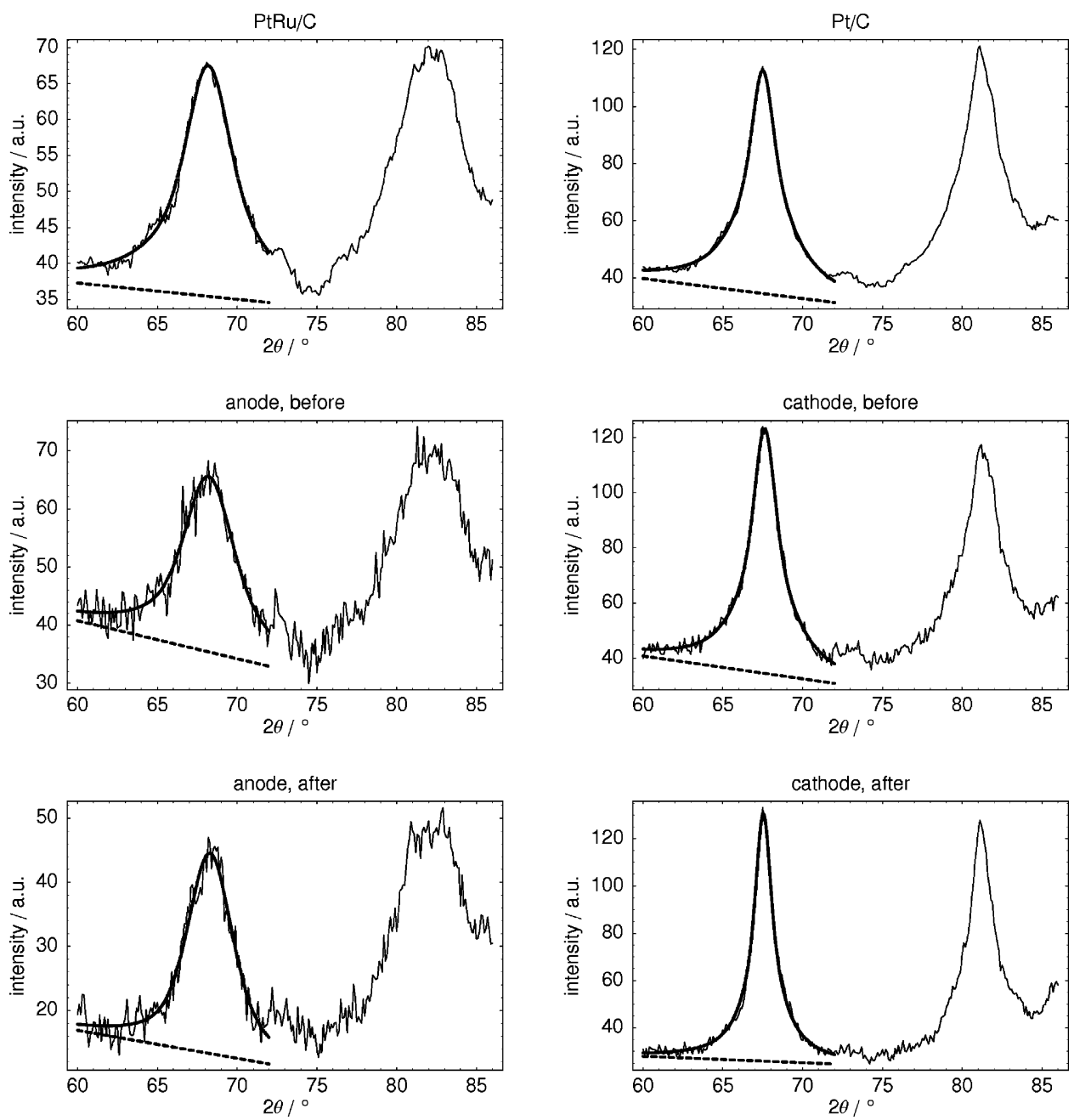

Figure 6. Diffraction data of the unused catalyst powders (first row), data extracted from the MEA before operation in the fuel cell environment (second row), and data extracted from the MEA after the $2100 \mathrm{~h}$ experiment. The thick lines represent least-square fits of pseudo-Voigt functions to the (220) reflections in the range from $2 \theta=60^{\circ}$ to $72^{\circ}$. The dashed lines show the linearly approximated diffraction background.

TABLE 2: Integral Breadths $\left(\boldsymbol{\beta}_{\mathrm{g}}, \boldsymbol{\beta}_{1}\right)$, Ratio of Volume-Weighted to Area-Weighted Mean Column Lengths $\left(\langle L\rangle_{\mathrm{v}} /\langle L\rangle_{\mathrm{s}}\right)$, Mean Particle Sizes Derived from That Data $\left(\langle P\rangle_{\text {xrd }}\right)$ and from TEM Images $\left(\langle P\rangle_{\text {tem }}\right)$, and Specific Surface Area $\left(S_{\mathrm{xrd}}\right)^{a}$

\begin{tabular}{llccccc}
\hline \multicolumn{1}{c}{ sample } & $\begin{array}{c}\beta_{\mathrm{g}} \\
\mathrm{nm}^{-1}\end{array}$ & $\begin{array}{c}\beta_{\mathrm{l}} \\
\mathrm{nm}^{-1}\end{array}$ & $\langle L\rangle_{\mathrm{v}} /\langle L\rangle_{\mathrm{s}}$ & $\begin{array}{c}\langle P\rangle_{\text {xrd }} \\
\mathrm{nm}\end{array}$ & $\begin{array}{c}\langle P\rangle_{\text {tem }} \\
\mathrm{nm}\end{array}$ & $\begin{array}{c}S_{\text {xrd }} \\
\mathrm{m}^{2} \mathrm{~g}^{-1}\end{array}$ \\
\hline $\mathrm{PtRu} / \mathrm{C}$ & 0.16 & 0.41 & 1.71 & 0.8 & 1.6 & 77 \\
$\mathrm{Pt} / \mathrm{C}$ & 0.077 & 0.27 & 1.81 & 1.1 & 1.6 & 50 \\
anode, before & 0.18 & 0.39 & 1.62 & 0.9 & 1.6 & 73 \\
cathode, before & 0.073 & 0.24 & 1.79 & 1.2 & 1.6 & 45 \\
anode, after & 0.18 & 0.29 & 1.47 & 1.5 & 2.1 & 55 \\
cathode, after & 0.052 & 0.16 & 1.77 & 1.8 & 2.1 & 31
\end{tabular}

${ }^{a}$ All values except for $\langle P\rangle_{\text {tem }}$ result from the fits of pseudo-Voigt functions to the diffraction profiles shown in Figure 6.

TABLE 3: Relative Changes of the Mean Crystallite Diameters $\left(\boldsymbol{\Delta}\langle\boldsymbol{P}\rangle_{\mathrm{xrd}}\right)$, Mean Particle Diameters $\left(\boldsymbol{\Delta}\langle\boldsymbol{P}\rangle_{\mathrm{tem}}\right)$, and Specific Surface Areas $\left(\Delta S_{\mathrm{xrd}}\right)^{a}$

\begin{tabular}{lccc}
\hline sample & $\Delta\langle P\rangle_{\mathrm{xrd}} / \%$ & $\Delta\langle P\rangle_{\mathrm{tem}} / \%$ & $\Delta S_{\mathrm{xrd}} / \%$ \\
\hline anode & 61 & 30 & -25 \\
cathode & 48 & 33 & -32
\end{tabular}

${ }^{a}$ The results are based on Table 3 comparing the absolute values before and after operating the MEA.

a statistical relevant analysis, and the results may not be directly comparable. For example, spatial variations in aging can arise from an inhomogeneous current distribution in the electrode.
$\mathrm{X}$-ray line profile analysis can alleviate these restrictions but needs some refinement with respect to the separation of the component signals if, as desired, the MEA is to be examined without alteration. The surface area covered by the X-ray beam is $1.6 \times 10^{8}$ times larger than that of a $100 \times 100 \mathrm{~nm}^{2}$ microscope image, and in addition, the entire thickness of the electrode contributes. Compared to the thin layer imaged by the microscope, the number of detected particles is about 10 orders of magnitude higher when using X-ray diffraction.

To obtain the particle size distributions from the diffraction patterns, a simplified Voigt function is fitted to the (220) peak and the result is used to calculate the parameters of a lognormal distribution. ${ }^{10}$ It is assumed that a particle with a diameter of only a few nanometers consists, due to the relatively high grain boundary energy, of only one coherently diffracting domain. However, it is important to carefully consider the results obtained from the analysis of line profiles. Physical broadening of the analyzed peak occurs due to lattice imperfections, like the finite size of the coherently diffracting domains and lattice strain. Methods are available, for example by Williamson and Hall, ${ }^{11}$ to separate the strain contribution from size-induced broadening. Previous studies indicate, however, that the strain component is negligible for particles with dimensions of only a few nanometers. For example, Scardi and Leoni find a strain component in nanocrystalline ceria that is close to the detection limit. ${ }^{12}$ Also Langford et al. report a very small strain-induced 

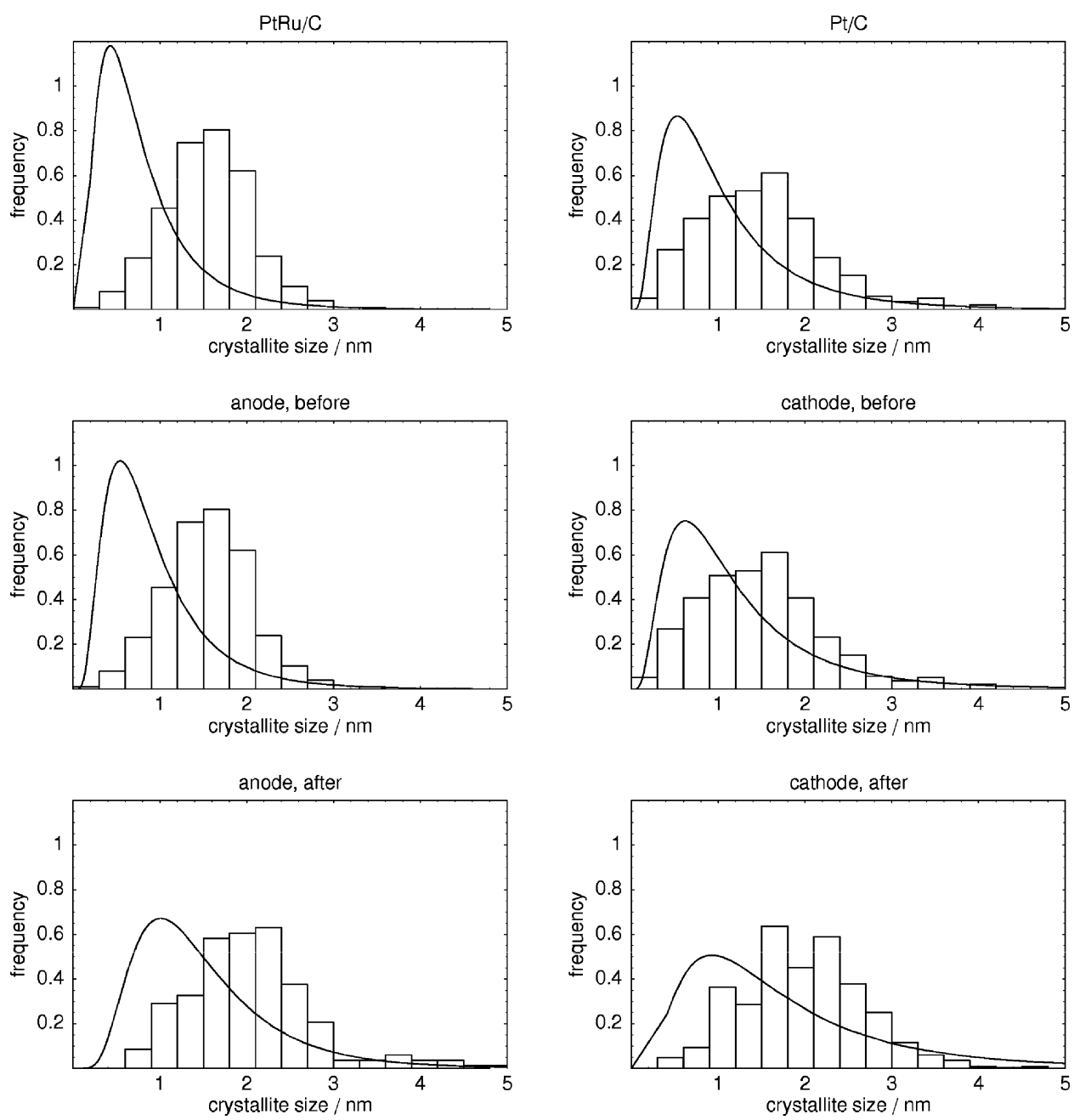

Figure 7. Comparison of the particle size distributions (see eq 12) derived from the parameters of the pseudo-Voigt functions fitted to the (220) diffraction peaks (continuous lines) with the size distributions obtained from TEM images (bar charts). To allow the comparison with the XRD distributions, the TEM data in the first and second rows are identical (see text for details). All distributions are normalized.

TABLE 4: Integral Breadths $\left(\beta_{\mathrm{g}}, \beta_{1}\right)$, Ratio of Volume-Weighted to Area-Weighted Mean Column Lengths $\left(\langle L\rangle_{\mathrm{v}} /\langle L\rangle_{\mathrm{s}}\right)$, the Mean Particle Sizes $\left(\langle P\rangle_{\mathrm{xrd}}\right)$, and the Specific Surface Areas $\left(S_{\mathrm{xrd}}\right)^{a}$

\begin{tabular}{llcccc}
\hline \multicolumn{1}{c}{ sample } & $\begin{array}{c}\beta_{\mathrm{g}} \\
\mathrm{nm}^{-1}\end{array}$ & $\begin{array}{c}\beta_{1} \\
\mathrm{~nm}^{-1}\end{array}$ & $\langle L\rangle_{\mathrm{v}} /\langle L\rangle_{\mathrm{s}}$ & $\begin{array}{c}\langle P\rangle_{\text {xrd }} \\
\mathrm{nm}\end{array}$ & $\begin{array}{c}S_{\text {xrd }} \\
\mathrm{m}^{2} \mathrm{~g}^{-1}\end{array}$ \\
\hline anode, $\mathrm{Ar}$ & 0.15 & 0.30 & 1.57 & 1.3 & 56 \\
cathode, $\mathrm{Ar}$ & 0.069 & 0.20 & 1.73 & 1.6 & 36 \\
anode, $\mathrm{H}_{2}$ & 0.16 & 0.26 & 1.49 & 1.6 & 49 \\
cathode, $\mathrm{H}_{2}$ & 0.067 & 0.18 & 1.71 & 1.8 & 34 \\
anode, $\mathrm{O}_{2}$ & 0.14 & 0.23 & 1.46 & 2.0 & 42 \\
cathode, $\mathrm{O}_{2}$ & 0.062 & 0.19 & 1.77 & 1.6 & 36
\end{tabular}

${ }^{a}$ All values are derived from the pseudo-Voigt functions fitted to the (220) peak.

TABLE 5: Changes of the Specific Surface Areas $\left(\Delta S_{\mathrm{xrd}}\right)$ after Storage for $6500 \mathrm{~h}$ in Each Gas Atmosphere Relative to the Values before Operation ${ }^{a}$

\begin{tabular}{lcc}
\hline gas & anode/\% & cathode/\% \\
\hline argon & -23 & -19 \\
hydrogen & -32 & -25 \\
oxygen & -42 & -19
\end{tabular}

${ }^{a}$ Compare to Table 4.

broadening, amounting to less than $1 \%$ of the total peak width. ${ }^{13}$ The present evaluation is therefore limited to the analysis of the size-induced broadening of the platinum (220) reflection. In this way, the negligible strain contribution remains uncon- sidered, but errors from analyzing other peaks that overlap with their neighbors are avoided. In spite of this minor error, the particle sizes show a considerable deviation from those found in the transmission electron micrographs. Historically, this observation has been made in many previous studies, even in analytical-model independent approaches, such as WarrenAverbach analysis, ${ }^{15}$ etc. For example, size deviations of $20 \%$ and $32 \%$ have been reported. ${ }^{12,13}$ Plausible causes of these size deviations are grain substructures with low-angle boundaries that are invisible to the microscope, lattice deformations on the surface of nanometer-sized particles, and small particles that are invisible in the micrographs due to sizes close to or below the resolution limit. Specific to the present experiments is the overlap of the analyzed (220) peak with a small PTFE peak (see above) that also induces a small error toward smaller particle sizes. To assess the individual contributions of the mentioned effects, further experiments are needed, especially with respect to the analysis of nanocrystalline particle systems.

Apart from their absolute values, the size distributions derived from the line profiles agree well with those obtained from the micrographs. After the operation in a fuel cell, a notable growth process occurs in which the distribution broadens and its maximum shifts toward larger sizes. Qualitatively, this result agrees well with earlier studies. For example Wilson et al. ${ }^{13}$ conducted a $4000 \mathrm{~h}$ long-term test and used line profile analysis to study the particle growth. The electrode material was removed from the MEA, dispersed in alcohol, and ultrasonicated before 
examination. Particle sizes were derived from the platinum (111) peak using the program supplied with the diffractometer that offered a Fourier analysis according to the method by Warren and Averbach. ${ }^{15}$ Although the analysis was complicated by the overlap of the size-broadened platinum (111) and (200) peaks, the results indicated an increase of the particle sizes and a loss of surface area on both electrodes, slightly more pronounced on the cathode. This agrees qualitatively with the loss of surface area that was observed in the present work, also being slightly more pronounced on the cathode.

Recently, another analysis of particle growth in polymer electrolyte fuel cells was performed by Ferreira. ${ }^{16}$ Two short fuel cell stacks were operated under similar conditions for 2000 $\mathrm{h}$ at open circuit voltage and at $0.2 \mathrm{~A} / \mathrm{cm}^{2}$ while the surface area of the cathode was checked periodically by evaluating the hydrogen adsorption charges in the recorded cyclic voltammograms. The respective losses of specific surface areas were found to be $80 \%$ and $50 \%$, although the voltage degradation was similar for both stacks at approximately $20 \mu \mathrm{V} / \mathrm{h}$. These electrochemically determined specific surface areas are not directly comparable with those measured by X-ray diffraction. Only ionically and electronically connected surfaces contribute in the electrochemical measurements, while line profile analysis provides an overview of all particles independent of their environment. For example, particles mobile on the carbon substrate that lose connection to the electrolyte or material dissolving and recrystallizing at unconnected particles is reported lost in terms of the electrochemically active surface. The contacted surface area also varies with many operating parameters of the fuell cell such as the hydration of the electrolyte, the temperature, etc. The surface area loss determined by electrochemical measurements is therefore higher than what is found by X-ray diffraction and represents a more transient state of the fuel cell, since the lost contact surface can potentially be reconnected under changed operating conditions. We believe that the actual loss of catalyst material as determined by X-ray diffraction provides more accurate information about the nonreversible performance degradation, as the surface lost by growth cannot be regained.

The MEA fragments that were stored in different gas atmospheres, studied in the second part of this work, also exhibit a notable loss of specific surface areas. Because the work functions of bulk platinum and bulk platinum-ruthenium are very similar, ${ }^{17}$ we assume that the potential difference between the anode and the cathode is small enough to be neglected. Therefore, and due to the very dry environment in the glass cylinders, electrochemical reactions are expected to be absent. Still, the measured surface area loss was approximately onethird of that in the operating fuel cell when based on the exposure time.

The particle growth is usually attributed to two different mechanisms. First, the agglomeration of individual particles that are mobile on their support, fusing once they are sufficiently close to each other. Second, the transport of metal ions from larger to smaller particles by dissolution and redeposition. Although the dissolution rate of bulk platinum is almost zero in the absence of an electrochemical potential, this might be different for nanometer-sized particles where a dissolution was found even though the potential was close to $0 \mathrm{~V}$ vs RHE. ${ }^{16}$ However, due to the low potential difference and the very low humidity in the glass cylinders, the contribution of the dissolution-redeposition process is probably small. We assume therefore that the main reason for the observed growth is particle agglomeration, a process that is further promoted by the high mobility of the metal particles on the carbon support. The lowest loss of surface area is found in argon and is higher, especially on the anode, in hydrogen and oxygen gases. This can be explained by the different surface energies of the particles in the gases, for example, by building up metal oxides on the surfaces. Because a small particle has a high surface-to-volume ratio, the influence of the surrounding atmosphere is substantial. For example, the gas in the environment of a small particle can even influence its shape ${ }^{18}$ and its composition on the surface. ${ }^{19}$ It is, however, not entirely clear why the platinum-ruthenium particles on the anode are more susceptible to growth than the platinum particles on the cathode. The surface loss during the fuel cell operation is approximately 2 times higher and, in contrast, more pronounced on the cathode $(32 \%)$ than on the anode $(25 \%)$. That is, the dissolution-redeposition process obviously plays a major role under operating conditions. Because of the potential-dependency of the platinum dissolution rate, the loss is higher on the cathode at a potential between 0.7 and $0.95 \mathrm{~V}$ vs RHE than on the anode at approximately $0 \mathrm{~V}$ vs RHE. Also, the carbon support is oxidized at the relatively high potential on the cathode, resulting in a higher particle mobility that promotes the agglomeration.

The repeated voltage cycling that was investigated in other studies, e.g., by Ferreira et. al, ${ }^{16}$ led to a considerably higher surface area loss on the cathode than during normal operation. Possibly, this is a consequence of the continuous formation and reduction of a surface oxide layer and the associated siteexchange reaction on the surface. For polycrystalline platinum, this place exchange occurs at approximately $1.1 \mathrm{~V}$ vs RHE 20 and an oxide film starts to form at approximately $0.85 \mathrm{~V}$ vs RHE. There is also evidence that this process starts already at lower potentials if the particles have dimensions on the nanometer scale. ${ }^{21}$

\section{Conclusion}

A technique was developed that allows the separation of individual components from a composite diffraction pattern. After extraction of the catalyst signals of the anode and the cathode from the diffraction patterns of an entire MEA, the line profile analysis provided the particle size distributions of the catalyst particles and the total surface area. To demonstrate the validity of this method, two identical MEAs were produced, and one was operated in a model PEMFC while the other was exposed to gases typically used during operation. By comparing particle sizes and surface areas before and after the experiments, we found a significant surface loss during the operation that was similar to the nonreversible voltage degradation under constant current operation. Although the same trend was observed with transmission electron microscopy, the particle sizes derived from the line profiles were significantly smaller than those in the micrographs. The electrodes of the identical MEA exposed to different gases showed approximately onethird of the surface loss, suggesting that the particle growth is not only induced by fuel cell operation. The presented technique allows the separation of diffraction patterns from multicomponent systems and is useful to study the changes of particle sizes and surface areas with a high statistical relevance and without destroying the specimen. It also provides a valuable foundation for future "in situ" experiments.

Acknowledgment. This work was financially supported by grants from the Forschungsallianz Brennstoffzelle BadenWürttemberg and the Deutsche Forschungsgemeinschaft. 


\section{References and Notes} 255 .

(1) Sankar, G.; Thomas, J. M.; Catlow, C. R. A. Top. Catal. 2000, 10,

(2) Roth, C.; Goetz, M.; Fuess, H. J. Appl. Electrochem. 2001, 31, 793.

(3) Viswanathan, R.; Hou, G. Y.; Liu, R. X.; Bare, S. R.; Modica, F.; Mickelson, G.; Segre, C. U.; Leyarovska, N.; Smotkin, E. S. J. Phys. Chem. B 2002, 106, 3458 .

(4) Roth, C.; Martz, N.; Buhrmester, T.; Scherer, J.; Fuess, H. Phys. Chem. Chem. Phys. 2002, 4, 3555.

(5) Roth, C.; Benker, N.; Buhrmester, T.; Mazurek, M.; Loster, M.; Fuess, H.; Koningsberger, D. C.; Ramaker, D. E. J. Am. Chem. Soc. 2005, 127,14607

(6) Arico, A. S.; Creti, P.; Kim, H.; Mantegna, R.; Giordano, N.; Antonucci, V. J. Electrochem. Soc. 1996, 143, 3950. 315.

(7) Roth, C.; Martz, N.; Fuess, H. Phys. Chem. Chem. Phys. 2001, 3,

(8) Thompson, P.; Cox, D. E.; Hastings, J. B. J. Appl. Crystallogr. 1987, 20, 79 .

(9) Balzar, D. Voigt-Function Model in Diffraction Line-Broadening Analysis. In Defect and Microstructure Analysis by Diffraction; Snyder,
R. L., Fiala, J., Bunge, H.J., Eds.; International Union of Crystallography: Chester, England, 1999; p 94.

(10) Popa, N. C.; Balzar, D. J. Appl. Crystallogr. 2002, 35, 338.

(11) Williamson, G. K.; Hall, W. H. Acta. Metall. 1953, 1, 22.

(12) Scardi, P.; Leoni, M. J. Appl. Crystallogr. 2006, 39, 24.

(13) Langford, J. I.; Louer, D.; Scardi, P. J. Appl. Crystallogr. 2000, 33,964 .

(14) Wilson, M. S.; Garzon, F. H.; Sickafus, K. E.; Gottesfeld, S. J. Electrochem. Soc. 1993, 140, 2872.

(15) Warren, B. E.; Averbach, B. L. J. Appl. Phys. 1950, 21, 595.

(16) Ferreira, P. J.; la O', G. J.; Shao-Horn, Y.; Morgan, D.; Makharia, R.; Kocha, S.; Gasteiger, H. A. J. Electrochem. Soc. 2005, 152, A2256.

(17) Bouwman, R.; Sachtler, W. M. H. J. Catal. 1972, 26, 63.

(18) Hansen, P. L.; Wagner, J. B.; Helveg, S.; Rostrup-Nielsen, J. R.; Clausen, B. S.; Topsoe, H. Science 2002, 295, 2053.

(19) Nashner, M. S.; Frenkel, A. I.; Adler, D. L., Shapley J. R.; Nuzzo, R. G. J. Am. Chem. Soc. 1997, 119, 7760.

(20) Jerkiewicz, G.; Vatankhah, G.; Lessard, J.; Soriaga, M. P.; Park, Y. S. Electrochim. Acta 2004, 49, 1451.

(21) Loster, M.; Friedrich, K. A.; Scherson, D. A. J. Phys. Chem. B 2006, 110, 18081 . 